syndromes, has recently been reported in a family with atypical Panyiotopoulos syndrome (Grosso S et al. Neurology 2007;69:609-611).

\title{
GOURMAND SYNDROME IN A CHILD WITH EPILEPSY
}

A preoccupation with food, increased appetite and a preference for fine food had developed in a 10-year-old obese boy soon after the onset of refractory seizures at 8 years of age, in a report from University Hospital of Geneva, Switzerland. He had streptococcal B sepsis and hemorrhage in the right temporoparietal lobes during the neonatal period. Seizures were stereotyped, with loss of contact and oral automatisms or dystonic posturing of the left hand. Their frequency was once a week to 7 times a day. They were uncontrolled despite trial of 5 different antiepileptic drugs. He preferred to cook meals for himself rather than eat at fast food restaurants. He had no history of emotional disorder, binge eating, bulimia, or preoccupation with his weight. Neurological examination revealed a left inferior quadrantanopia and left dysdiadochokinesia. In presurgical evaluation, the EEG showed a right posterior focus, and diffuse bilateral parasaggital seizure activity in sleep. MRI revealed a porencephaly, periventricular gliosis and hemosiderin deposits in the right parietal lobe. Neuropsychological examination showed visuospatial memory deficits, discrete signs of neglect, more pronounced in the postictal phase, with perseveration and confabulation. (Kurian M, Schmitt-Mechelke T, Korff C, Delavelle J, Landis T, Seeck M. "Gourmand syndrome" in a child with pharmacoresistant epilepsy. Epilepsy Behav August 2008;13:413415). (Respond: Dr M Kurian, Presurgical Epilepsy Evaluation Unit, Department of Neurology, University Hospital of Geneva, 24 rue Micheli-du-Crest, 1211 Geneva 14, Switzerland. E-mail: mary.kurian(ahcuge.ch).

COMMENT. "Gourmand" syndrome" reported in adults with right hemisphere lesions is often associated with epilepsy, and the eating disorder may be reversible when epilepsy is controlled. (Levine R et al. Epilepsy Behav 2003;4:781-3; cited by Kurian et al). The authors recommend brain imaging to rule out right hemisphere lesion in a patient who develops disturbed eating habits following a head injury or seizures.

\section{RISK OF DROWNING IN EPILEPSY}

The risk of drowning in patients with epilepsy is quantified by a meta-analysis of published reports, in a study at the Institute of Neurology, Queen Square, London UK. The number of deaths from drowning and the number of person-years at risk were estimated in 51 cohorts of people with epilepsy. Standardized mortality ratios (SMRs) (observed deaths divided by the expected deaths) were calculated for each cohort and for the total population. Compared with 4.7 expected deaths, 88 drowning deaths were reported in people with epilepsy, giving an SMR of 18.7 (95\% Cl 15-23). The 51 cohorts combined had 206,596 patient-years of follow-up. In people with epilepsy and learning disability, the SMR was 25.7, and in those in institutional care, 96.9. In those with temporal lobectomy for epilepsy, the SMR was 41.1. Using National Registries for estimation of drowning deaths in people with epilepsy in England and Wales (1999-2000), the SMR was 15.3. (Bell GS, Gaitatzis A, Bell CL, Johnson AL, Sander JW. Drowning in people with epilepsy. How great is the risk. Neurology Aug 19 2008;71:578-582). (Reprints: Prof Ley Sander, Box 29, Department of 
Clinical \& Experimental Epilepsy, UCL Institute of Neurology, Queen Square, London WC1N 3BG, UK. E-mail: Isander(a) ion.ucl.ac.uk).

COMMENT. Compared to the general population, people with epilepsy have a 15- to 19- fold increase in risk of drowning, according to this meta-analysis of published articles. The actual number of drownings in children is small, only 4 of the 15 articles involving predominantly children including deaths due to drowning. Inadequate supervision was a frequent factor. One previous study suggested that the relative risk of drowning in the bath is 96 for children with epilepsy compared to those without, and for drowning in a swimming pool is 23 (Diekema DS et al. Pediatrics 1993;91:612-616). One in 20 deaths from drowning was due to seizures (Ryan CA et al. CMAJ 1993;148:781-784).

\section{GENETICS OF AUTOSOMAL DOMINANT PARTIAL EPILEPSY WITH AUDITORY FEATURES (ADPEAF)}

Data from 24 previously published ADPEAF families with mutations in the leucinerich, glioma inactivated 1 gene (LGI1) were analyzed, in a study at Columbia University, New York. Penetrance is $67 \%$, it tends to be greater in families with more affected individuals, does not differ with gender, and may increase with advancing generation. (Rosanoff MJ, Ottman R. Penetrance of LGI1 mutations in autosomal dominant partial epilepsy with auditory features. Neurology Aug 19, 2008;71:567-571). (Dr Ruth Ottman, GH Sergievsky Center, Columbia Univcrsity, $630 \mathrm{~W} 168^{\text {th }}$ St, P\&S Box 16, New York, NY 10032. E-mail: ro6(a)columbia.edu).

COMMENT. Autosomal dominant (AD) partial epilepsy with auditory features, also known as AD lateral temporal epilepsy, is an idiopathic focal epilepsy syndrome manifested by auditory symptoms (humming, buzzing, or ringing, volume changes, specific songs or voices) or receptive aphasia. Ictal receptive aphasia is a sudden inability to understand language without general confusion. Mutations in the LGII gene located on chromosome 10 occur in approximately $50 \%$ of families with ADPEAF, but not in families with other familial temporal lobe epilepsies.

Symptomatic temporal lobe auditory seizures. Many examples of seizures secondary to temporal lobe tumor and manifested by auditory illusions and hallucinations are included in the classic book by Penfield W, and Jasper H (Epilepsy and the Functional Anatomy of the Human Brain. Boston; Little, Brown \& Comp. 1954;459-467). One patient, a housewife of 43 years, complained of ringing in the ears and of hearing voices or music, a song she had heard previously, an hallucination sometimes she described as a dream. Another, a girl aged 16 years, heard a lullaby that her mother used to sing to her as an infant. In some attacks she complained of a change in volume or fading away of sounds, considered an illusional seizure. Stimulation of the cortex in the superior gyrus of the left temporal lobe illicited hallucinations of voices, saying words, repeated music (accompanied by humming), and "There was singing" response. Penfield referred to these as "psychical seizures." In children, temporal lobe seizures often begin with a sense and look of fear, the child clinging to mother, although unable to describe a possible associated hallucination. 